\title{
Associações entre Macroinvertebrados e Peixes Demersais na Armação do Itapocoroy, Penha, Sc, Brasil.
}

\author{
Joaquim Olinto Branco ${ }^{1,2 *}$, Maria José Lunardon-Branco ${ }^{2}$, Alberto Carvalho Peret ${ }^{3}$, \\ Flávio Xavier Souto ${ }^{1}$, Rodrigo Schveitzer ${ }^{1}$ and Willian Guimarães Vale ${ }^{1}$. \\ ${ }^{1}$ - Faculdade de Ciências do Mar - FACIMAR / UNIVALI. CX. Postal 360, 88301-970, Itajaí, SC. ${ }^{2}$-Programa de \\ Pós-Graduação em Ecologia e Recursos Naturais, UFSCar. ${ }^{3}$-Universidade Federal de São Carlos -UFSCar, \\ Depto de Hidrobiologia. Cx. Postal 676, 13565-905, São Carlos, SP.
}

\begin{abstract}
In the period of January and December, 1995, there were collected by means of overtrawl, 12977 samples of macroinvertebrates and demersal fishes, in the Armação do Itapocoroy, Penha (SC), between the depths of 6 to 10 meters, in the periods of morning, afternoon, and evening. There were registered the incidence of 57 families, 80 kinds and 92 species distributed between the macroinvertebrates and demersal fishes. The families of greater incidence were: Portunidae (30.7\%), Strombidae (11.4\%), Sciaenidae (11.1\%) and Bothidae (10.3\%) which all together, contributed with $63.5 \%$ of the total samples. The grouping analysis presented greater diversity in the Winter (2.77) and smaller one in the Springtime $(1,34)$; the amplitude of variation $H^{\prime}$ reflects the great number of species of both occasional and seasonal in the Armação do Itapocoroy.
\end{abstract}

Key Words: Association, macroinvertebrates, demersal fishes, Armação do Itapocoroy

\section{INTRODUÇÃO}

Este trabalho faz parte de um projeto mais amplo, em desenvolvimento no litoral Catarinense, voltado para a caracterização da pesca artesanal do camarão-sete-barbas (Xiphopenaeus kroyeri), e de suas relações com a fauna acompanhante.

A participação de peixes, crustáceos, moluscos, equinodermatas e cnidários na composição da fauna associada à captura de camarões, efetuada através de redes de arrastos com portas é elevada, superando consideravelmente a biomassa de camarões em condições de comercialização (Coelho et al., 1986).

O conhecimento desse valioso recurso é de fundamental importância, visto que a pesca de arrasto é considerada predatória e desestabilizadora das comunidades bentônicas, sendo frequentemente realizadas em criadouros de espécies de interesse econômico (Ruffino \& Castello, 1992/93).
$\mathrm{O}$ presente trabalho tem como objetivo estabelecer as associações faunísticas entre os turnos de coleta, bem como observar o comportamento da diversidade de macroinvertebrados e peixes demersais na pesca do camarão-sete-barbas na Armação do Itapocoroy, Penha (SC).

\section{MATERIAL E MÉTODOS}

Durante o período de janeiro/95 a dezembro/95 foram realizadas coletas mensais nos turnos da manhã, tarde e noite, na Armação do Itapocoroy $\left(26^{\circ} 46^{\prime}-26^{\circ} 47^{\prime} \mathrm{S}\right)$ e $\left(48^{\circ} 36^{\prime}-48^{\circ} 37^{\prime} \mathrm{W}\right)$. A profundidade na área de coleta variou entre 6 a 10 metros, sendo o fundo biodetrítico.

As capturas foram efetuadas através de duas redes-de-arrasto com portas, cada uma com 45 metros de cabo, 6 metros de abertura de boca, malha de 3,0 cm na manga e no corpo e $2,0 \mathrm{~cm}$ no ensacador. $\mathrm{O}$ tempo de cada arrasto foi de 30 minutos e a velocidade média da embarcação foi de 2 nós.

*Autor para correspodência 
Os Crustacea Decapoda foram identificados de acordo com Williams (1984), Holthuis (1980) e Hebling \& Rieger (1986). Os Mollusca segundo Rios (1975); os Echinodermata de acordo com Tommasi (1970) e os Perciformes em base de Figueiredo (1977), Fischer (1978), Figueiredo \& Menezes $(1978 ; 1980)$ e Menezes \& Figueiredo (1980).

As espécies foram classificadas, segundo sua ocorrência nas coletas, em três categorias (Ansari et al., 1995): regular (9 a 12 meses); sazonal (6 a 8 meses) e ocasional (1 a 5 meses). Para o cálculo da diversidade foram utilizados três índices considerados mais informativos: índice de riqueza específica de Margalef (D), índice de diversidade de Shannon $\left(\mathrm{H}^{\prime}\right)$ e índice de equitabilidade de Pielou (J'); foram calculados mensalmente, conforme Ludwig \& Reynolds (1988).

Através da análise de agrupamento, foram estabelecidas as associações entre as espécies e os turnos das coletas, utilizando-se o número de indivíduos por espécie. Devido à distribuição contagiosa típica de macroinvertebrados e peixes (Colvocoresses \& Musick, 1984), procedeu-se a padronização dos dados com a transformação $\operatorname{logarítmica~} \log (\mathrm{x}+1)$. Na sequência, foi estabelecida a medida de semelhança entre os pares de espécies pelo coeficiente de distância Euclidiana (melhor coeficiente cofenético) e agrupados, pelo método UPGMA (Unweighted Pair Group Method-Averages). Foram eliminadas das análises de agrupamento as espécies que ocorreram em menos de 3 coletas por turno.

\section{RESULTADOS E DISCUSSÃO}

Ocorrência: Foram capturados 12977 exemplares entre macroinvertebrados e peixes demersais, pertencentes a 92 espécies, 80 gêneros e 57 famílias em 36 arrastos entre os períodos da manhã, tarde e noite, durante o ano de 1995 (Tab.I). Destas, 52 espécies ocorreram nos três períodos. A maior diversidade específica foi durante a noite, com 76 espécies, enquanto que os demais períodos contribuíram, cada um, com 68 espécies.
A maior abundância numérica ocorreu durante à tarde, com 4598 exemplares; à noite com 4380 exemplares e pela manhã com 3999 exemplares (Tab.I).

A família Portunidae foi a de maior ocorrência $(30,7 \%)$, seguida pelas famílias Strombidae $(11,4 \%)$, Sciaenidae $(11,1 \%)$ e Bothidae $(10,3 \%)$. As quatro famílias, em conjunto, contribuíram com $63,5 \%$ do total de exemplares capturados.

Abundância e densidade: As variações sazonais na abundância e densidade dos macroinvertebrados e peixes demersais, nos três períodos, durante o ano de 1995, são mostradas nas Fig. 1a e 1b.

No mês de abril foi registrada a maior abundância de espécies com queda em maio, oscilando entre os períodos até agosto, decrescendo gradativamente até novembro (Fig.1a).

A densidade relativa em número de indivíduos apresentou padrão de variação distinto entre os períodos de coleta. A maior frequência de captura ocorreu durante a tarde no mês de abril; à noite em maio, pela manhã em outubro (Fig.1b).

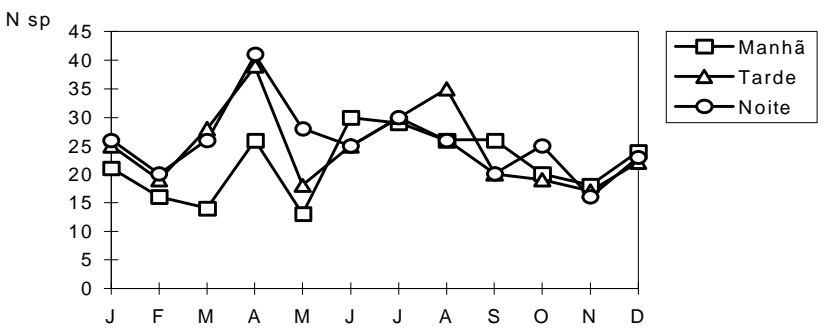

$\mathrm{N}$

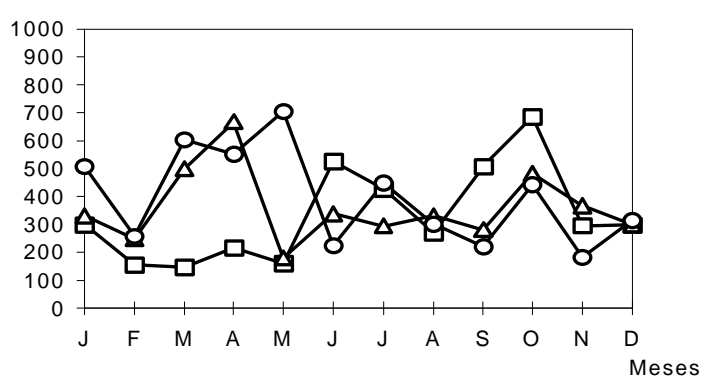

Fig. 1. Frequência mensal do número de espécies (a) e indivíduos (b) capturados durante o ano de 1995 na Armação do Itapocoroy. 
Análise das associações: Para a análise das associações foram analisadas 39 espécies no período da manhã, 41 espécies na tarde e 35 espécies na noite; 27 espécies foram comuns aos três períodos

No período da manhã, a análise de agrupamento destaca a formação de 5 grupos faunísticos (Fig. 2): O grupo I, formado por Cymatyum parthenopeum e Prionotus punctatus, de ocorrência ocasional e sazonal, respectivamente (Tab.I). O grupo II apresentou o maior agrupamento, com 15 espécies representadas por Mollusca, Crustacea, Echinodermata e Perciformes, que contribuíram com 172 exemplares. Dessas espécies, 14 foram ocasionais e apenas Siratus senegalensis foi sazonal (Tab. I). O grupo III composto por 191 exemplares, pertencentes a 6 espécies, 2 foram ocasionais, 3 sazonais e Synodus foetens de ocorrência regular, contribuiu com $0,8 \%$ do total coletado no período (Tab. II). O grupo IV formado por 5 espécies ocasionais $\mathrm{e}$ representando 281 exemplares (Tab. I). O grupo $\mathrm{V}$ apresentou a maior abundância numérica, com 1220 exemplares representando 30,4\% do período. Das 6 espécies do grupo V (Tab. II) apenas Penaeus paulensis foi sazonal, às demais foram regulares.

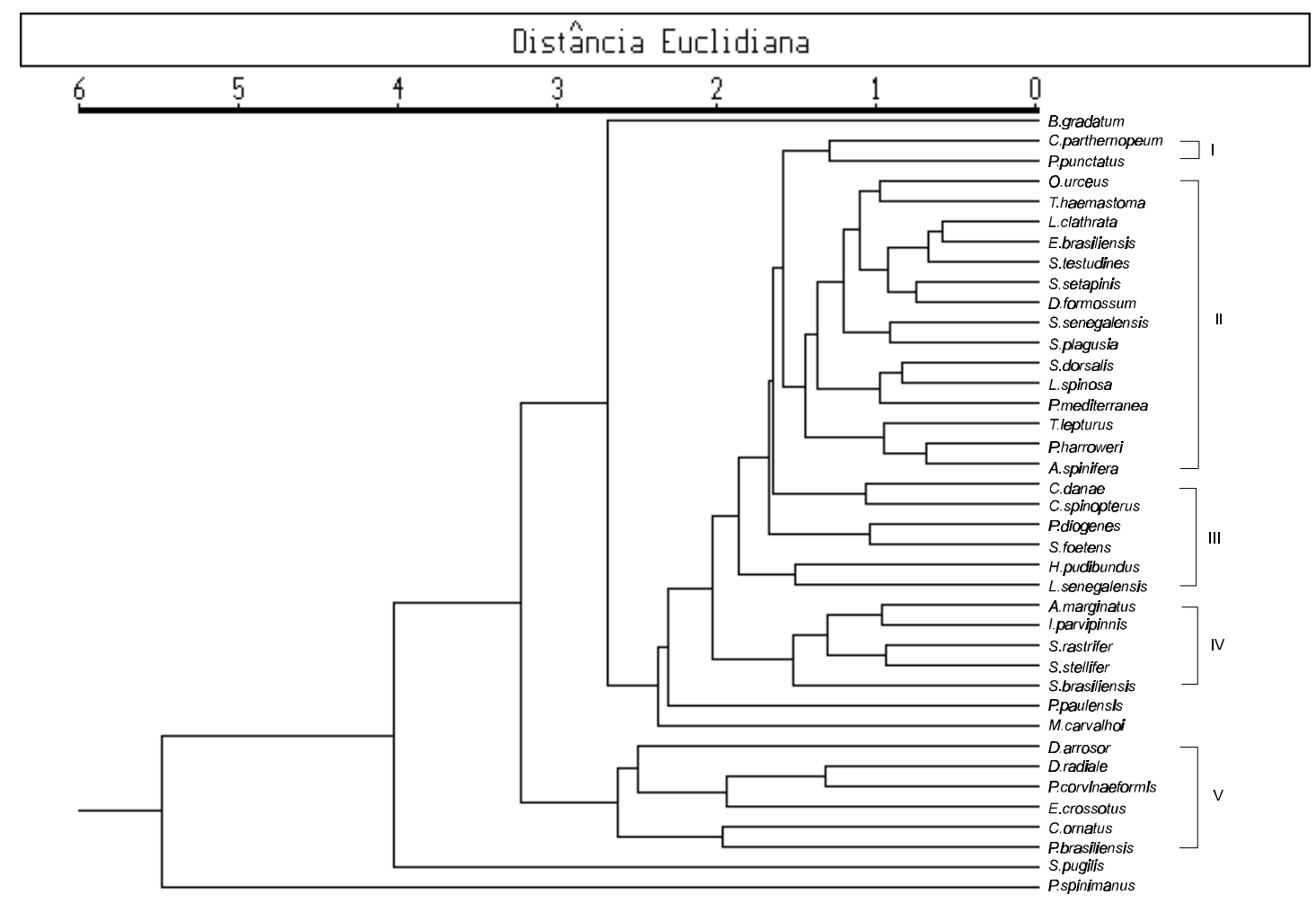

Fig. 2. Dendrograma mostrando o agrupamento das espécies de macroinvertebrados e peixes demersais da manhã.

Os Mollusca Gastropoda Buccinanops gradatum e Strombus pugilis, de ocorrência sazonal, participaram com 92 e 562 exemplares, respectivamente, perfazendo em conjunto, $16,3 \%$ do total de exemplares capturados no período da manhã; Portunus spinimanus com 1063 exemplares e ocorrência regular representou $26,6 \%$ do período (Tab. I e II). Embora, com essa participação, não foram incluídos nos grupos (Fig. 2). 
Tab. I. Relação das espécies de macroinvertebrados e peixes demersais e suas respectivas frequências por período de coleta, na Armação do Itapocoroy durante o ano de 1995. A ocorrência das espécies nas coletas é representada por $(>=$ regular; $+=$ sazonal; < = ocasional).

\begin{tabular}{|c|c|c|c|c|c|c|c|}
\hline $\begin{array}{r}\text { Família/Espécies } \\
\end{array}$ & Manhã & Ocor. & Tarde & Ocor. & Noite & Ocor. & Anual \\
\hline \multicolumn{8}{|l|}{ Mollusca/ Gastropoda } \\
\hline \multicolumn{8}{|l|}{ Trochidadae } \\
\hline Tegula viridula (Gmelin, 1791) & - & - & 1 & $<$ & - & - & 1 \\
\hline \multicolumn{8}{|l|}{ Turbinidae } \\
\hline Astrea latispina (Philippi, 1844) & - & - & - & - & 2 & $<$ & 2 \\
\hline \multicolumn{8}{|l|}{ Cymatiidae } \\
\hline Cymatyum parthenopeum (von Salis, 1793) & 29 & $<$ & 49 & + & 71 & + & 149 \\
\hline \multicolumn{8}{|l|}{ Muricidae } \\
\hline Siratus senegalensis (Gmelin, 1970) & 18 & + & 4 & $<$ & 7 & $<$ & 29 \\
\hline \multicolumn{8}{|l|}{ Traididae } \\
\hline Thais haemostoma (Linnaeus, 1767) & 17 & $<$ & 20 & $<$ & 65 & + & 102 \\
\hline \multicolumn{8}{|l|}{ Nassariidae } \\
\hline \multicolumn{8}{|l|}{ Strombidae } \\
\hline Strombus pugilis (Linnaeus, 1822) & 562 & + & 633 & $>$ & 285 & + & 1480 \\
\hline \multicolumn{8}{|l|}{ Olividae } \\
\hline Olivacillaria urceus (Röding, 1798) & 8 & $<$ & 172 & + & 2 & $<$ & 182 \\
\hline \multicolumn{8}{|l|}{ Mollusca/ Bivalvia } \\
\hline Anadara brasiliana (Lamarck, 1819) & - & - & 4 & $<$ & - & - & 4 \\
\hline \multicolumn{8}{|l|}{ Mytilidae } \\
\hline $\begin{array}{l}\text { Modiolus carvalhoi (Klappenbach, 1966) } \\
\text { Cardiidae }\end{array}$ & 81 & $<$ & 14 & \multicolumn{3}{|c|}{ Cardiidae } & 95 \\
\hline Trachycardium muricatum (Linnaeus, 1758) & 3 & $<$ & - & - & - & - & 3 \\
\hline \multicolumn{8}{|l|}{ Veneridae } \\
\hline Chione cancellata (Linnaeus, 1767) & 2 & $<$ & - & - & - & - & 2 \\
\hline Pitar aresta (Dall \& Simpson, 1900) & 3 & $<$ & 6 & $<$ & 3 & $<$ & 12 \\
\hline \multicolumn{8}{|l|}{ Tellinidae } \\
\hline \multirow{2}{*}{\multicolumn{8}{|c|}{ Mollusca/ Cephalopoda }} \\
\hline & & & & & & & \\
\hline \multicolumn{8}{|l|}{ Loligonidae } \\
\hline Lolliguncula brevis (Brainville, 1823) & 7 & $<$ & 2 & $<$ & 6 & $<$ & 15 \\
\hline Loligo sanpaulensis (Brakoniecki, 1984) & - & - & 1 & $<$ & - & - & 1 \\
\hline \multicolumn{8}{|l|}{ Crustacea/ Malacostraca } \\
\hline Penaeus paulensis (Pérez Farfante, 1967) & 75 & + & 112 & $>$ & 273 & $>$ & 460 \\
\hline Penaeus schmitti (Burkenroad, 1936) & 1 & $<$ & 2 & $<$ & 3 & $<$ & 6 \\
\hline \multicolumn{8}{|l|}{ Solenoceridae } \\
\hline Pleoticus mиelleri (Bate, 1888) & - & - & - & - & 5 & $<$ & 5 \\
\hline \multicolumn{8}{|l|}{ Diogenidae } \\
\hline \multicolumn{7}{|l|}{ Calappidae } & 938 \\
\hline Hepatus pudibundus (Herbst, 1785) & 36 & $<$ & 48 & $>$ & 114 & + & 198 \\
\hline Leucossidae & & & & & & & \\
\hline Persephona mediterranea (Herbst, 1794) & 27 & $<$ & - & - & 1 & $<$ & 28 \\
\hline Persephona punctata (Linnaeus, 1758) & 12 & $<$ & 19 & $<$ & 22 & $<$ & 53 \\
\hline Majidae & & & & & & & \\
\hline Libinia spinosa (H. Milne Edwards, 1834) & 6 & $<$ & - & - & 2 & $<$ & 8 \\
\hline Portunidae & & & & & & & \\
\hline Callinectes danae (Smith, 1869) & 40 & $<$ & - & - & 2 & $<$ & 42 \\
\hline Callinectes ornatus (Ordway, 1863) & 287 & $>$ & 263 & $>$ & 477 & $>$ & 1027 \\
\hline Portunus spinimanus (Latreille, 1819) & 1065 & $>$ & 823 & $>$ & 1026 & $>$ & 2914 \\
\hline
\end{tabular}


Continuação Tab. I.

\begin{tabular}{|c|c|c|c|c|c|c|c|}
\hline Família/Espécies & Manhã & Ocor. & Tarde & 0cor. & Noite & Ocor. & Anual \\
\hline Cronius ruber (Lamarck, 1818) & 1 & $<$ & - & - & 2 & $<$ & 3 \\
\hline Arenaeus cribarius (Lamarck, 1818) & 10 & $<$ & 4 & $<$ & - & - & 14 \\
\hline \multicolumn{8}{|l|}{ Xanthidae } \\
\hline Pilumnus dasypodus (Kingley, 1879) & 1 & $<$ & 2 & $<$ & - & - & 3 \\
\hline \multicolumn{8}{|l|}{ Echinodermata/ Asteroidea } \\
\hline \multicolumn{7}{|l|}{ Luidiidae } & 35 \\
\hline Luidia senegalensis (Lamarck, 1816) & 38 & + & 22 & + & 45 & + & 105 \\
\hline \multicolumn{8}{|l|}{ Asterinidae } \\
\hline $\begin{array}{l}\text { Asterina stellifera (Tortonese, 1962) } \\
\text { Echinasteridae }\end{array}$ & \multicolumn{6}{|c|}{ Echinasteridae } & 8 \\
\hline $\begin{array}{l}\text { Echinaster brasiliensis (Müller \& Troschel, 1842) } \\
\text { Perciformes/ Chondrichthyes } \\
\text { Rhinobatidae }\end{array}$ & 5 & $<$ & 9 & $<$ & 13 & + & 27 \\
\hline \multicolumn{8}{|l|}{ Narcinidae } \\
\hline $\begin{array}{l}\text { Narcine brasiliensis (Olfwea, 1831) } \\
\text { Perciformes/ Osteichthyes } \\
\text { Muraenidae }\end{array}$ & 3 & $<$ & 3 & $<$ & 4 & $<$ & 10 \\
\hline \multicolumn{8}{|l|}{ Ophichtidae } \\
\hline $\begin{array}{l}\text { Ophichtus gomesì (Castelnau, 1855) } \\
\text { Congridae }\end{array}$ & \multicolumn{6}{|c|}{ Congridae } & 15 \\
\hline \multicolumn{8}{|l|}{ Clupeidae } \\
\hline Pellona harroweri (Fowler, 1917) & 20 & $<$ & 75 & $<$ & 41 & $<$ & 136 \\
\hline Harengula clupeola (Cuvier, 1829) & 1 & $<$ & - & - & 3 & $<$ & 4 \\
\hline Chirocentrodus brakerianus (Poey, 1867) & 2 & $<$ & - & - & - & - & 2 \\
\hline \multicolumn{8}{|l|}{ Engraulidae } \\
\hline Cetengraulis edentulus (Cuvier, 1829) & - & - & - & - & 1 & $<$ & 1 \\
\hline \multicolumn{7}{|l|}{ Synodontidae } & 21 \\
\hline \multicolumn{7}{|l|}{ Batrachoididae } & 91 \\
\hline \multicolumn{8}{|l|}{ Ogcocephalidae } \\
\hline \multicolumn{8}{|l|}{ Scorpaenidae } \\
\hline Scorpaena brasiliensis (Cuvier, 1829) & - & - & - & - & 1 & $<$ & 1 \\
\hline \multicolumn{7}{|l|}{ Triglidae } & 8 \\
\hline \multicolumn{8}{|l|}{ Centropomidae } \\
\hline Centropomus paralelus (Poey, 1860) & - & - & - & - & 1 & $<$ & 1 \\
\hline Diplectrum formossum (Linnaeus, 1758) & 7 & $<$ & 63 & + & 40 & + & 110 \\
\hline Mycteroperca rubra (Bloch, 1793) & - & - & - & - & 4 & $<$ & 4 \\
\hline Epinephelus niveatus (Valenciennes, 1828) & - & - & - & - & 1 & $<$ & 1 \\
\hline \multicolumn{8}{|l|}{ Carangidae } \\
\hline Oligoplites saurus (Bloch \& Schneider, 1801) & 2 & $<$ & - & - & 1 & $<$ & 3 \\
\hline Chloroscombus chrysurus (Linnaeus, 1766) & 1 & $<$ & 6 & $<$ & - & - & 7 \\
\hline Selene setapinnis (Mitchill, 1815) & 5 & $<$ & 9 & $<$ & 1 & $<$ & 15 \\
\hline Selene vomer (Linnaeus, 1758) & - & - & 1 & $<$ & - & - & 1 \\
\hline \multicolumn{8}{|l|}{ Gerreidae } \\
\hline Eugerres brasilianus (Cuvier, 1830) & - & - & 2 & $<$ & 3 & $<$ & 5 \\
\hline Eucinostomus argenteus (Baird \& Girard, 1854) & - & - & 3 & $<$ & 2 & $<$ & 5 \\
\hline Eucinostomus melanopterus (Bleeker, 1863) & - & - & 1 & $<$ & - & - & 1 \\
\hline
\end{tabular}




\begin{tabular}{|c|c|c|c|c|c|c|c|}
\hline Família/Espécies & Manhã & Ocor. & Tarde & 0cor. & Noite & Ocor. & Anual \\
\hline Eucinostomus gula (Cuvier, 1830) & - & - & - & - & 1 & $<$ & 1 \\
\hline \multicolumn{8}{|l|}{ Pomadasyidae (Haemulidae) } \\
\hline Pomadasys corvinaeformis (Steindachner,1868) & 128 & $>$ & 188 & $>$ & 132 & $>$ & 448 \\
\hline Anisotremus virginicus (Linnaeus, 1758) & - & - & 1 & $<$ & - & - & 1 \\
\hline \multicolumn{8}{|l|}{ Sciaenidae } \\
\hline Larimus breviceps (Cuvier, 1830) & 10 & $<$ & 1 & $<$ & 1 & $<$ & 12 \\
\hline Isopisthus parvipinnis (Cuvier, 1830) & 65 & $<$ & 68 & $<$ & 74 & $>$ & 207 \\
\hline Paralonchurus brasiliensis (Steindachner, 1875) & 113 & + & 174 & + & 136 & $>$ & 423 \\
\hline Stellifer rastrifer (Jordan, 1889) & 57 & $<$ & 36 & $<$ & 68 & $<$ & 161 \\
\hline Stellifer stellifer (Bloch, 1790) & 38 & $<$ & 13 & $<$ & 32 & + & 83 \\
\hline Steliifer brasiliensis (Schultz, 1945) & 94 & $<$ & 346 & $<$ & 102 & + & 542 \\
\hline Micropogonias furnieri (Desmarest, 1823) & - & - & 4 & $<$ & 2 & $<$ & 6 \\
\hline Menticirrhus americanus (Linnaeus, 1758) & 3 & $<$ & 4 & $<$ & 4 & $<$ & 11 \\
\hline Menticirrhus littoralis (Holbrook, 1860) & - & - & - & - & 1 & $<$ & 1 \\
\hline \multicolumn{8}{|l|}{ Pomacanthidae } \\
\hline Pomacanthus paru (Bloch, 1787) & 2 & $<$ & 3 & $<$ & 1 & $<$ & 6 \\
\hline Peprilus paru (Linnaeus, 1758) & - & - & - & - & 1 & $<$ & 1 \\
\hline \multicolumn{8}{|l|}{ Trichiuridae } \\
\hline Trichiurus lepturus (Linnaeus, 1758) & 16 & $<$ & 20 & + & 3 & $<$ & 39 \\
\hline \multicolumn{8}{|l|}{ Bothidae } \\
\hline Etropus crossotus (Jordan \& Gilbert, 1881) & 290 & $>$ & 490 & $>$ & 503 & $>$ & 1283 \\
\hline Citharichthys spilopterus (Gunther, 1862) & 19 & + & 25 & + & 13 & $<$ & 57 \\
\hline \multicolumn{8}{|l|}{ Soleidae } \\
\hline Achirus lineatus (Linnaeus, 1758) & - & - & 1 & $<$ & - & - & 1 \\
\hline \multicolumn{8}{|l|}{ Cynoglossidae } \\
\hline Symphurus plagusia (Linnaeus, 1766) & 10 & $<$ & 11 & $<$ & 36 & $<$ & 57 \\
\hline \multicolumn{8}{|l|}{ Monocanthidae } \\
\hline Stephanolepis hispidus (Linnaeus, 1766) & - & - & - & - & 3 & $<$ & 3 \\
\hline \multicolumn{8}{|l|}{ Tetraodontidae } \\
\hline Lagocephalus laevigatus (Linnaeus, 1766) & 1 & $<$ & 9 & $<$ & 3 & $<$ & 13 \\
\hline Sphaeroides testudineus (Linnaeus, 1758) & 4 & $<$ & 2 & $<$ & 5 & $<$ & 11 \\
\hline \multicolumn{8}{|l|}{ Diodontidae } \\
\hline Chilomycterus spinosus (Linnaeus, 1758) & 3 & $<$ & 4 & $<$ & 1 & $<$ & 8 \\
\hline Total & 3999 & & 4598 & & 4380 & & 12977 \\
\hline
\end{tabular}

À tarde, formou-se 5 grupos (Fig. 3): o grupo I composto por 5 espécies, com 140 exemplares, sendo 2 ocasionais, 2 sazonais e Synodus foetens regular (Tab. I e II). No grupo II ocorreu o maior agrupamento do período com 16 espécies oriundas da associação entre macroinvertebrados e peixes demersais que contribuíram com 138 exemplares, desta, 14 espécies foram ocasionais e os Echinodermata Luidia senegalensis $e L$. clathrata foram sazonais (Tab. I). O grupo III formado pelas espécies Hepatus pudibundus e Prionotus punctatus com ocorrência regular e sazonal, respectivamente, participaram com 74 exemplares. O grupo IV composto por 7 espécies, destas, 4 foram ocasionais e 3 sazonais, contribuindo com 642 exemplares (Tab. I).
$\mathrm{O}$ grupo $\mathrm{V}$, formado por 8 espécies com ocorrência regular nas coletas, contribuiu com 2852 exemplares, representando $62,0 \%$ do total de exemplares capturados no período da tarde (Tab. II).

À noite, assim como nos demais períodos, houve a formação de 5 grupos (Fig. 4): o grupo I participou com 298 exemplares pertencentes a 5 espécies. Destas, uma foi ocasional, 3 sazonais e Isopisthus parvipinnis de ocorrência regular (Tab. I e II). O grupo II formado por 16 espécies destacou-se como o maior agrupamento do período, sendo representado por 271 exemplares. Cinco espécies foram de ocorrência sazonal e 11 ocasionais. 


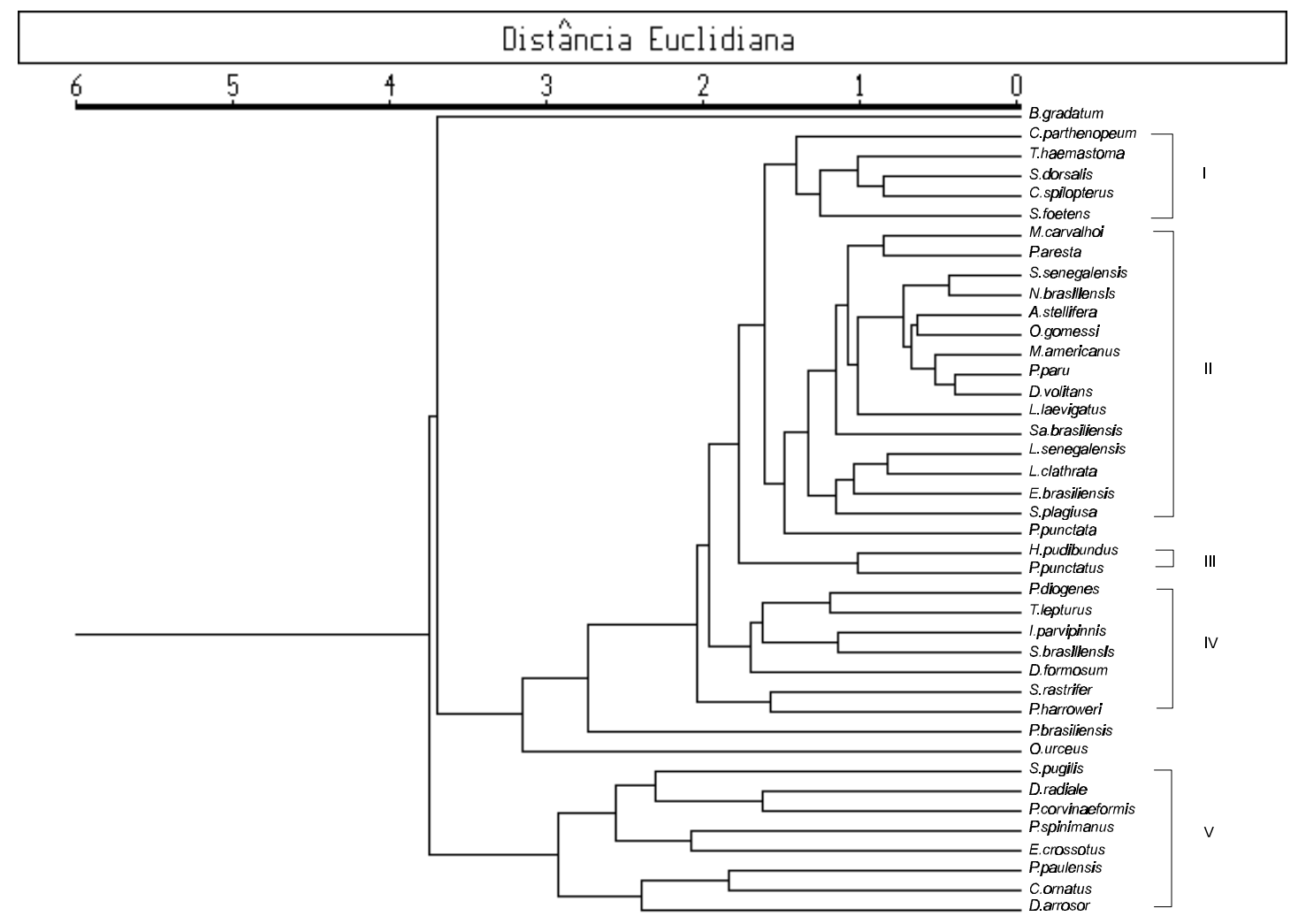

Fig. 3. Dendrograma mostrando o agrupamento das espécies de macroinvertebrados e peixes demersais da tarde.

O grupo III, composto por 5 espécies com 364 exemplares, sendo 1 espécie ocasional, 3 sazonais e Petrochirus diogenes de ocorrência regular. $\mathrm{O}$ grupo IV, formado por 4 espécies de ocorrência regular, participaram com 660 exemplares, perfazendo $15,0 \%$ do período (Tab. II). O grupo V, composto por 5 espécies que contribuíram em conjunto, com 2682 exemplares. Neste grupo, apenas o Gastropoda Strombus pugilis foi de ocorrência sazonal, as 4 espécies regulares representaram $54,7 \%$ do total de exemplares coletados durante à noite (Tab. II).

A análise da associação entre comunidades de macroinvertebrados e peixes demersais não são frequentes na literatura. Em geral, são capturados em conjunto nos arrastos de fundo mas abordados isoladamente, conforme o interesse e a especialização do pesquisador. Assim, diferentes metodologias são utilizadas pelos autores e as variações na composição das associações de outras áreas, dificultam as comparações com os resultados obtidos na região da Armação do Itapocoroy Penha - SC.

As 16 espécies de Mollusca capturadas, representaram 19,3\% do número total de exemplares coletados, sendo que Strombus pugilis com $13,7 \%$ foi a única espécie regular, mas apenas durante a tarde.

As 17 espécies de Crustacea Decapoda contribuíram com 46,2\% do total de exemplares coletados. As espécies Portunus spinimanus, Callinectes ornatus, Dardanus insignis, apresentaram ocorrência regular durante o ano, independente do turno de amostragem. Enquanto que as cinco espécies de Echinodermata participaram com apenas $1,5 \%$ do total de exemplares coletados e não apresentaram espécies regulares.

Os peixes, com 54 espécies, representaram 33,0\% do número total de exemplares capturados; apenas Etropus crossotus, Pomadasys corvinaeformis e Diplectrum radiale foram regulares nos três turnos de amostragens.

Poucas espécies dominaram nos arrastos de fundo, em cada período. Durante o turno da manhã, 3 espécies de Crustacea e 4 de peixes, representaram $56,0 \%$ do total de exemplares coletados nesse período. $\mathrm{Na}$ tarde, uma espécie 


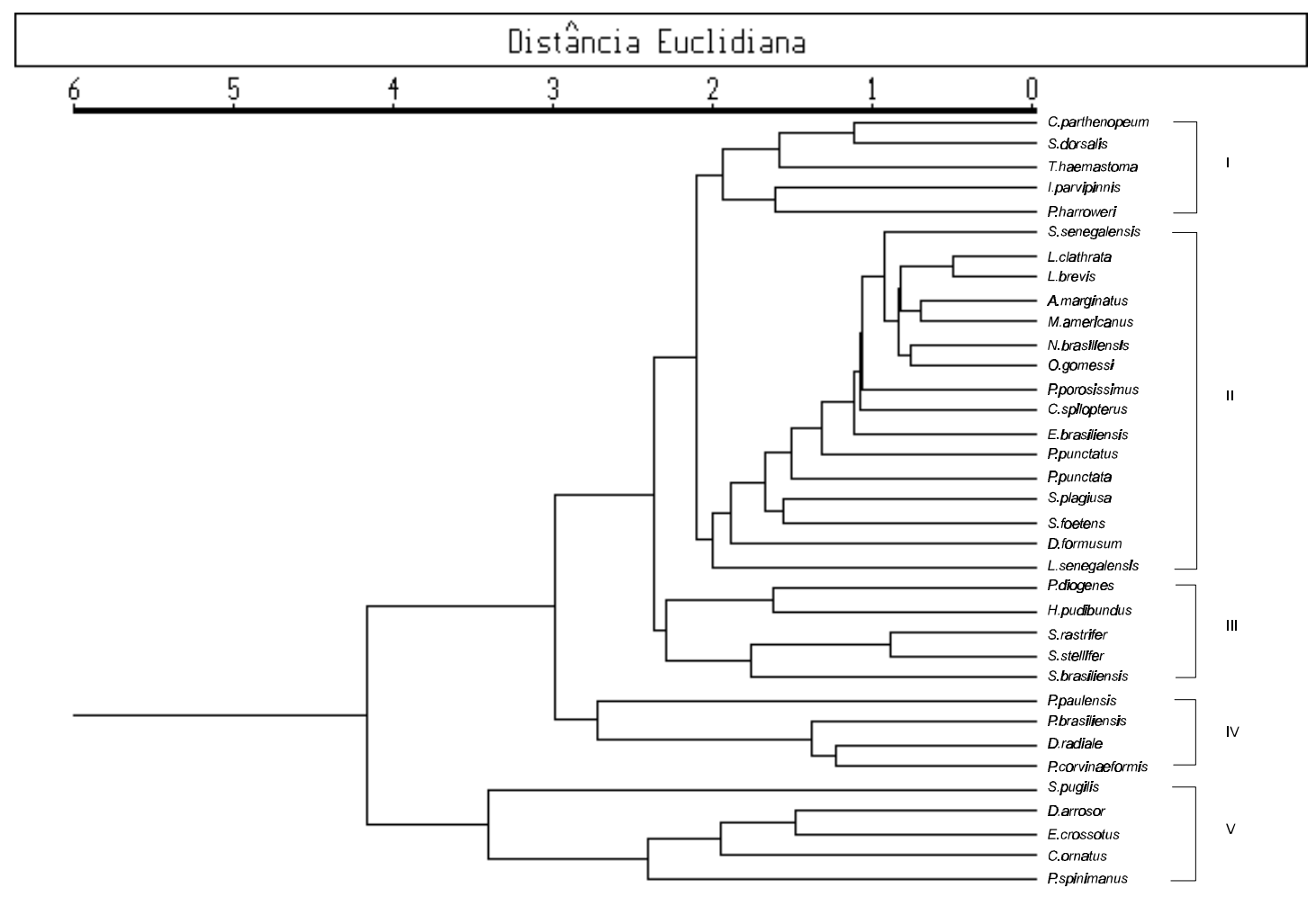

Fig. 4. Dendrograma mostrando o agrupamento das espécies de macroinvertebrados e peixes demersais da noite.

Tab. II. Relação das espécies regulares e suas respectivas porcentagens nos períodos de coleta durante o ano de 1995 na Armação do Itapocoroy.

\begin{tabular}{|c|c|c|c|}
\hline Família/ Espécie & Manhã & Tarde & Noite \\
\hline \multicolumn{4}{|l|}{ Strombidae } \\
\hline Strombus pugilis & - & 13.7 & - \\
\hline \multicolumn{4}{|l|}{ Penaeidae } \\
\hline $\begin{array}{l}\text { Penaeus paulensis } \\
\text { Diogenidae }\end{array}$ & - & 2.4 & 6.2 \\
\hline $\begin{array}{l}\text { Dardanus arrosor } \\
\text { insignis }\end{array}$ & 7.9 & 5.0 & 8.9 \\
\hline \multicolumn{4}{|l|}{ Paguridae } \\
\hline \multicolumn{3}{|l|}{ Calappidae } & 1.1 \\
\hline $\begin{array}{l}\text { Hepatus pudibundus } \\
\text { Portunidae }\end{array}$ & \multicolumn{2}{|c|}{ Portunidae } & - \\
\hline Callinectes ornatus & 7.2 & 5.7 & 10.9 \\
\hline \multicolumn{4}{|l|}{ Synodontidae } \\
\hline $\begin{array}{l}\text { Synodus foetens } \\
\text { Serranidae }\end{array}$ & \multicolumn{2}{|c|}{ Serranidae } & - \\
\hline \multicolumn{3}{|l|}{ Pomadasyidae } & 2.7 \\
\hline $\begin{array}{l}\text { Pomadasys } \\
\text { corvinaeformis }\end{array}$ & 3.2 & 4.2 & 3.0 \\
\hline \multicolumn{4}{|l|}{ Sciaenidae } \\
\hline $\begin{array}{l}\text { Isopisthus parvipinnis } \\
\text { Paralonchurus } \\
\text { brasiliensis }\end{array}$ & - & $\begin{array}{l}- \\
-\end{array}$ & $\begin{array}{l}1.7 \\
3.1\end{array}$ \\
\hline \multicolumn{4}{|l|}{ Bothidae } \\
\hline Etropus crossotus & 7.2 & 10.7 & 11.5 \\
\hline Total geral por período & 3999 & 4598 & 4380 \\
\hline
\end{tabular}

de Mollusca, 5 de Crustacea e 4 de peixes foram responsáveis por $63,3 \%$ dos exemplares coletados no período. Enquanto que na noite, 5 espécies de Crustacea e 5 de peixes, representaram $72,5 \%$ dos exemplares coletados.

Esse padrão de dominância observado na associação de macroinvertebrados e peixes demersais na Armação do Itapocoroy, confere com vários estudos de comunidades de peixes em região costeira (Fagundes Neto \& Caelzer, 1991), em estuário (Pereira, 1994) e laguna costeira (Monteiro-Neto et al., 1990).

As oscilações na abundância registradas nesse estudo, podem ser atribuídas ao padrão de ocorrência das espécies diurnas e noturnas, bem como, com a capacidade de evitar a redede-arrasto ou turbidez da água.

Para Mccleave \& Fried (1975), a capacidade dos peixes de maior porte em avistar e evitar a rede, é a responsável pela menor ocorrência nas coletas diurnas. 
Pereira (1994), trabalhando com peixes demersais na Lagoa dos Patos, RS, observou que espécies consideradas noturnas, ocorreram em maior número durante o dia.

Índices de diversidade: Os índices de riqueza específica de Margalef (D), de diversidade de Shannon (H') e de equitabilidade de Pielou (J') (Fig. 5a,b,c), apresentaram valores mais elevados no primeiro semestre do ano, diminuindo a partir de julho até o mês de setembro.

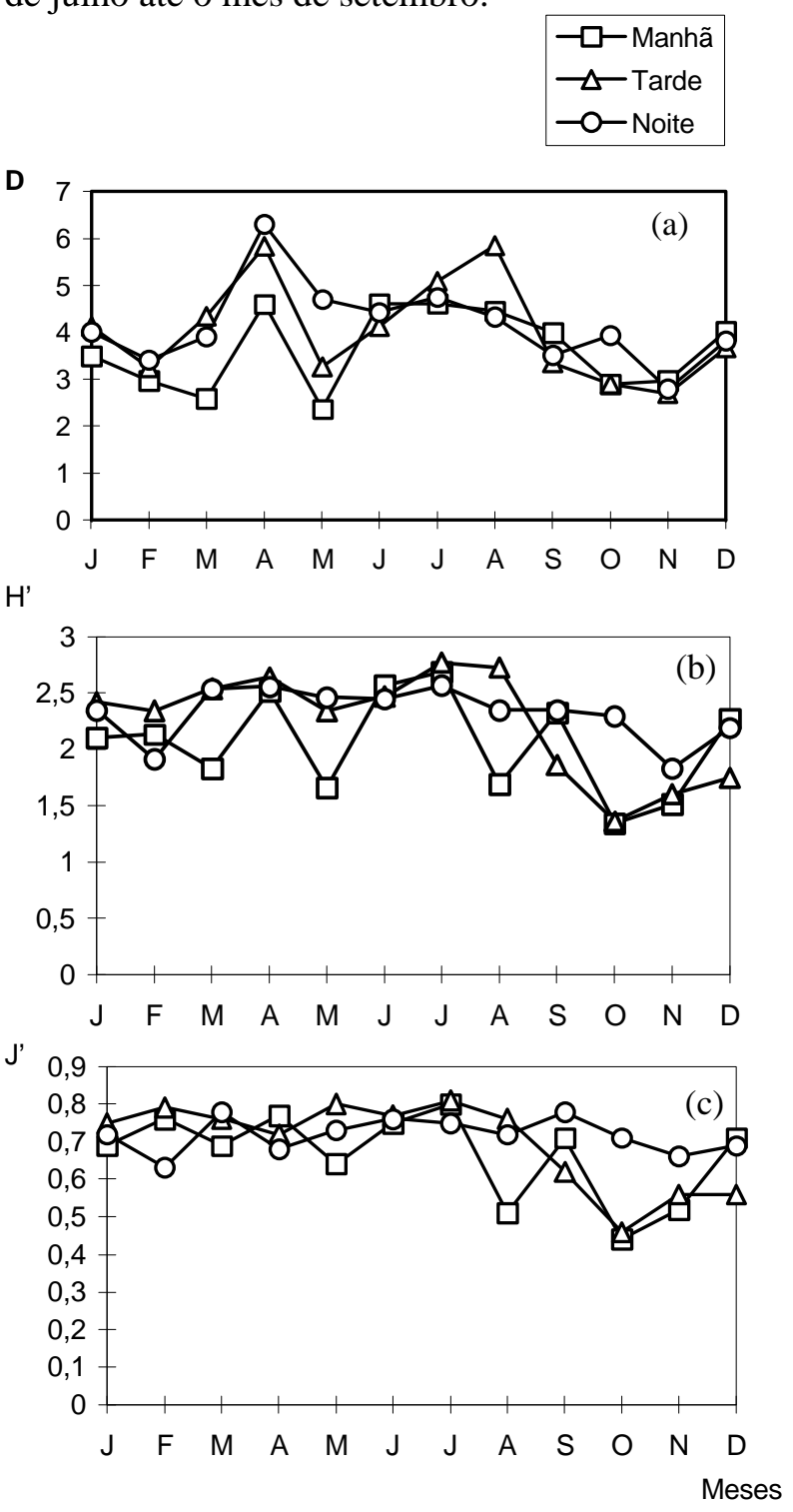

Fig. 5. Variação mensal dos índices de riqueza de espécies (D) (a), diversidade (H') (b) e equitabilidade (J') (c), durante o ano de 1995 na Armação do Itapocoroy.

$\mathrm{O}$ índice $\mathrm{D}$ variou mensalmente, mantendo o mesmo padrão entre os períodos, com o maior valor no mês de abril, oscilando até agosto, e queda gradativa até novembro (Fig. 5a).

O índice H' apresentou padrão semelhante de variação do índice $\mathrm{D}$, com valor máximo de 2,77 no inverno (julho, no período da tarde) e mínimo de 1,34 na primavera (outubro, manhã). De maneira geral, os valores mais elevados do verão foram registrados à tarde, enquanto que na primavera ocorreram durante à noite (Fig.5 b).

A equitabilidade (J') apresentou flutuação semelhante a H', porém com oscilações mais evidentes e diferenças marcantes entre os períodos (Fig. 5c).

$\mathrm{O}$ índice de riqueza de espécies, relativamente alto, com poucas espécies numericamente dominantes, aparentemente são características comuns em associações de peixes demersais (Ansari et al., 1995). Para Blaber et al. (1989), a alta diversidade e riqueza de espécies é um padrão marcante nos estuários subtropical e tropical do Indo-Pacífico.

A amplitude de variação do H' observado ao longo do ano entre os três períodos de coleta, refletem o grande número de espécies com ocorrência ocasional e sazonal, que utilizam a região da Armação do Itapocoroy, Penha, como local para alimentação, crescimento e reprodução.

\section{AGRADECIMENTOS}

À Pró-Reitoria de Pesquisa e PósGraduação da UNIVALI, pelo auxílio econômico e bolsa de iniciação científica PIBIC concedida a R. Schveitzer. Ao Diretor da FACIMAR e aos Professores e estagiários do Campus V, Penha, pelas facilidades colocadas a nossa disposição. Bem como, aos ex-estagiários do Laboratório de Oceanografia Biológica (Zoologia), acadêmicos: Paulo Afonso Brunetta, Cláudio Rogério Guerra, Hugo R. L. Diogo, Mario da Silva Freitas, Luciano Strefling, pelo auxílio nas coletas e processamento das amostras. 


\section{REFERÊNCIAS BIBLIOGRÁFICAS}

Ansari, Z. A.; A. Chatterfi; B. S. Ingole; R. A. Sreepada; C. U. Rivonkar \& A. H. Parulekar. (1995), Community structure and seasonal variation of an inshore demersal fish community at Goa, West Coast of India. Estuar. Coast. Shelf. Sci., 41:593-610.

Blaber, S. J. M.; D. T. Brewer \& J. P. Salini. (1989), Species composition and biomasses of fishes in differents habitats of a tropical northern Australian estuary: their ocurrence in the adjoining sea and stuarine dependence. Estuar. Coast. Shelf Sci., 29:509-531.

Coelho, J. A. P.; A. Puzzi; R. Graça-Lopes; E.S.Rodrigues \& O. Preto Jr.(1986), Análise da rejeição de peixes na pesca artesanal dirigida ao camarão sete-barbas (Xiphopenaeus kroyeri) no litoral de São Paulo. Bolm. Inst. Pesca, São Paulo, 13 (2): 51-61.

Colvocoresses, J. A. \& J. A. Musick. (1984), Species associations and community compositions of middle Atlantic Bight continental shelf demersal fishes. Fish. Bull., 82:295-313.

Fagundes Neto, E. B. \& Caelzer, L. R. (1991), Associações de peixes bentônicos e demersais na região de Cabo Frio, RJ, Brasil. Nerítica, Curitiba, (1-2):139-156.

Figueiredo, J. L. (1977), Manual de Peixes Marinhos do Sudeste do Brasil. I. Introdução Cações, raias e quimeras. Museu de Zoologia. USP. 104p.

Figueiredo, J. L. \& Menezes, N.(1978), Manual de Peixes Marinhos de Sudeste do Brasil. II. Teleostei (1). Museu de Zoologia. USP. 110p.

Figueiredo, J. L. \& Menezes, N. (1980), Manual de Peixes Marinhos do Sudeste do Brasil. III. Teleostei (2). Museu de Zoologia. USP. 90p.

Fischer, W. (ed.) (1978), FAO species identifications sheets for fishery purposes. Western Central Atlantic (Fishing area 31). v.1-7.

Hebling, N. J. \& Rieger, P.J. (1986), Os ermitões (Crustacea, Decapoda: Paguridae e Diogenidae) do litoral do Rio Grande do Sul, Brasil. Atlântica, Rio Grande, 8: 63-77.
Holthuis, L. B. (1980), Shrimp and praws of the world. An annoted catalogue of species of interest to fisheries. FAO Fish. Synop.,125(1):1-261.

Ludwig, J. A. \& Reynolds, J. F. (1988), Statistical ecology: a primer on methods and computing. John Wiley \& Sons, Inc. $338 \mathrm{p}$.

Menezes, N. \& Figueiredo, J. L. (1980), Manual de Peixes Marinhos do Sudeste do Brasil. IV. Teleostei (3) .Museu de Zoologia. USP. 96p

Mccleave, J. D. \& Fried, S. M. (1975), Nighttime catches of fishes in a tidal cove in Montsweag Bay near Wiscasset, Maine. Trans. Amer. Fish. Soc., n 1.

Monteiro-Neto, C.; Blacher, C. Laurent, A. A. S.; Snizek, F. N.; Canozzi, M. B. \& Tabajara, L. L. C. A. (1990), Estrutura da comunidade de peixes em águas rasas na região de Laguna, Santa Catarina, Brasil. Atlântica, Rio Grande, 12(2):53-69.

Pereira, L. E. (1994), Variação diurna e sazonal dos peixes demersais na barra do Estuário da Lagoa dos Patos, RS. Atlântica, Rio Grande, 16:5-21.

Rios, E. C. (1975), Brazilians Marine Mollusks Iconography. Fundação Universidade de Rio Grande. 1328p.

Ruffino, M. L. \& Castello, J. P. (1992/93), Alterações na ictiofauna acompanhante da pesca do camarão-barba-ruça (Artemesia longinaris) nas imediações da barra de Rio Grande, Rio Grande do Sul - Brasil. Nerítica, Curitiba, 7(1-2):43-55.

Tommasi, L. R. (1970), Lista dos Asteroides recentes do Brasil. Contrib. Inst. Oceanogr., sér. ocean. biol., (18):1:161.

Williams, A. B. (1984), Shrimps, lobsters and crabs of the Atlantic coast the Eastern United States Marine Florida. Smithsonian Institution Press. 550 p. 